\title{
A Smart Health Device to Measure Waist Circumfence
}

\author{
Jochen Meyer \\ OFFIS Institute for Information Technology \\ Oldenburg, Germany \\ meyer@offis.de
}

\author{
Timo Schoormann, Daniel Wegmann, \\ Ahmad Albuhasi \\ Carl von Ossietzky University Oldenburg \\ Oldenburg, Germany \\ [firstname.lastname]@uni-oldenburg.de
}

\begin{abstract}
Although the body mass index is a frequently used measure for overweight and obesity, the waist circumfence is a more reliable predictor for cardiovascular risks. However, while the first one can easily be digitally monitored using networked body scales there is no solution yet for the latter. We therefore present a smart measuring tape system that supports the user in taking consistent measures of the waist circumfence and stores the measures digitally. The system comprises a smartphone app and a digital measuring tape. An LED based interface helps the user in conducting the measurements. Users found the system easy to use and interesting.
\end{abstract}

Keywords—digital measuring tape; smart health devices

\section{INTRODUCTION}

In the past few years self-monitoring of health has received considerable attention. Smart health devices [1] are now available that are characterized by a) measuring determinants of health such as daily activity, sports, or sleep, b) are a combination of a physical device and an online service, and c) are by design easy to use consumer products for the mass market. One of the most established and most popular determinants of health is the body weight and the derived measure "body mass index" (BMI = weight in kilogram / (height in meter) ${ }^{\wedge} 2$ ). Usually a BMI in the range 18.5 to 25 is considered normal weight, 25-30 overweight and above 30 obese [2]. Networked scales such as the Withings Smart Body Analyzer or Fitbit Aria ${ }^{1}$ (as well as many traditional scales) calculate it based on input of the user's height.

However as a predictor for e.g. cardiovascular risks the BMI is not the only, let alone best easy measure. Rather the waist circumfence is at least an equally reliable or even better measure [3]. Waist cirumfence can easily be measured by laypersons: After breathing out a measuring tape is placed firmly midway between hip bone and bottom of rib. For women waist circumfence should be below $80 \mathrm{~cm}$, for men below $94 \mathrm{~cm}$.

To date there is no smart health device available for measuring the waist circumfence. We therefore developed the

\footnotetext{
${ }^{1}$ http://www.withings.com/smart-body-analyzer.html; http://www.fitbit.com/aria
}

prototype of a smart waist measuring tape that supports the user in taking correct and consistent measures and stores the measures digitally for review, analysis and long-term observation.

\section{APPROACH}

\section{A. Concept}

The smart measuring tape system comprises two components, the actual tape for taking the measure, and a smartphone app for storing, analyzing and presenting the measures. The two components communicate via Bluetooth.

For the sake of development effort we restricted the smartphone app to the basic functionality, communicating with the measuring tape and presenting the measurements (see figure 1). This is just enough to evaluate the functioning and usability of the overall system. In a real-life system more options could be implemented such as instructions and help for the user, multi-user support, forwarding the data to an internet service, reminders and encouragements etc.

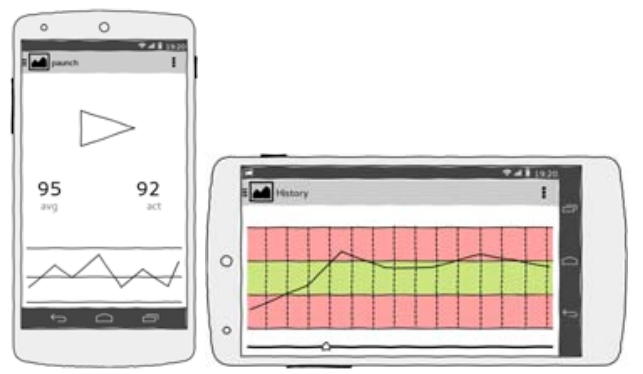

Fig. 1. The measuring app

\section{B. The measuring tape}

The measuring tape is built after the example of a boxed rollup measuring tape used e.g. in household and construction, see figure 2: The tape is rolled up around an axis within a closed housing. When being pulled out, a spring is extended that pulls back the tape when it is released. The spring also ensures that the tape lies tightly around the waist; this is important for consistent and correct measures. 


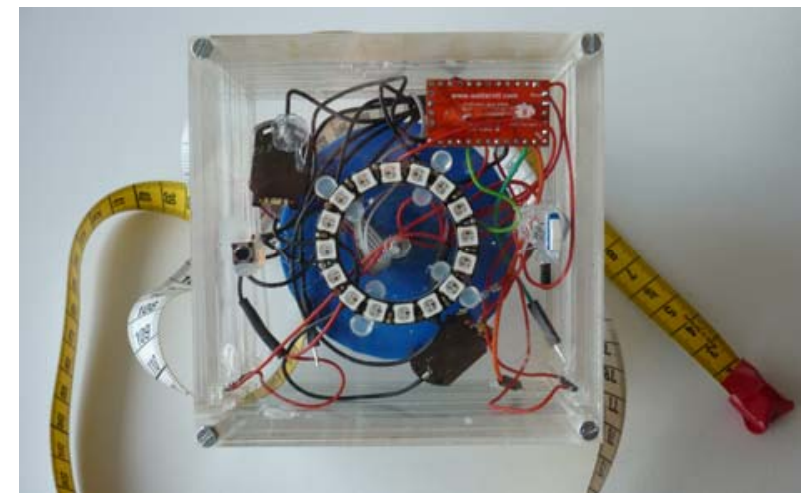

Fig. 2. The measuring tape

To conduct the measure the user pulls out the tape, lays it around its waist and closes the loop by touching the box with the end of the tape. The end tape is equipped with a magnet, and the touch is detected by a magnetic contact in the box (see Fig. 3, left). Before finalizing the measure the tape must be kept still in place for a few seconds to make sure that the measure is as consistent and correct as possible.

The measuring tape has a circular LED interface - as can be seen in figure 2 - to guide the user through the three stages of the measuring the process: When pulling out the tape the LEDs rotate red to indicate that the measure has started and the loop must be closed. When the loop is closed - as detected by the magnetic contact - the LEDs successively light up like a progress bar to indicate that the user must stay still for a few seconds. When the measure has successfully been taken the LEDs pulsate green. The user can now safely slide back the tape into the box.

The technical core of the measuring tape is an incremental encoder attached to the axis of the box (see figure 3, right) that measures how far the axis has been rotated. This can easily be related to the distance that the tape has been pulled off the box.
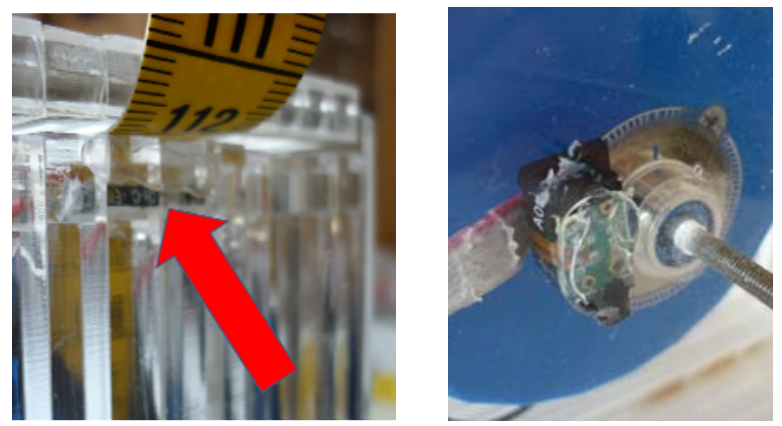

Fig. 3. Details: magnetic contact (left) incremental encoder (right)

\section{EVALUTION}

End users were involved as part of a user centered design process. An early version of the measurement tape was tested by a group of persons interested in weight lost and experienced in weight monitoring using scales. They found the measurement process easy to handle and the feedback from the LED circle good to understand. The final system was evaluated by 10 persons using the "AttracDiff" questionnaire [4]. In comparison to a conventional, analogue measuring tape the users found the smart one more interesting, creative and of value, but also technically more complicated and less predictable.

\section{DISCUSSION AND OUTLOOK}

We present a proof of concept for a new type of smart health device. Our current system is just a prototype that is still too bulky, not beautiful and reliable enough, and has technical issues. However the basic concept was well understood and appreciated by the end users. And we believe that the technical issues are due to implementation challenges, not because of bad design. Therefore we think that a more sophisticated version should be able to mitigate the users' critics.

It is an inherent property of the measurement method that the correct use cannot be guaranteed. The user could unintendedly take the measurement at the wrong position around the waist, or he could intentionally cheat by sucking his belly. However, when properly used the system improves the consistency of the measurement by ensuring a consistent pull of the tape using the spring mechanism.

The main advantage of the system, however, is the fact that the measurements are stored and processed digitally without further user action. Previously the only way to include waist circumfence in health application was a manual input by the user, which is prone to errors and too much effort in the long term. With the smart measuring tape the waist circumfence as a significant body measure can now seamlessly be included in applications such as long-term monitoring or weight loss interventions.

We showed that a digital measuring tape can technically be built using fairly simple electronics and mechanics and can easily and intuitively be used by laypersons. Future work could now examine the effects of monitoring the waist circumfence compared to the traditional method of weighing.

\section{References}

[1] Jochen Meyer, Susanne Boll. Smart Health Systems for Personal Health Action Plans. IEEE Healthcom'14 - 16th International Conference on Ehealth Networking, Application \& Services. Natal, Brazil. Nov 2014

[2] Pearson, T. A., Blair, S. N., Daniels, et al. (2002). AHA Guidelines for Primary Prevention of Cardiovascular Disease and Stroke. Circulation, (106), 388-391.

[3] Ian Janssen, Peter T Katzmarzyk, and Robert Ross. Waist circumference and not body mass index explains obesity-related health risk Am J Clin Nutr 2004 79: 3 379-384

[4] Hassenzahl, M., Burmester, M. \& Koller, F. (2003). AttrakDiff: Ein Fragebogen zur Messung wahrgenommener hedonischer und pragmatischer Qualität (A questionnaire for the measurement of percieved hedonicstic and pragmatic quality). In: J. Ziegler \& G. Szwillus (Hrsg.), Mensch \& Computer 2003. Interaktion in Bewegung (S. 187-196). Stuttgart, Leipzig: B.G. Teubner. 